\title{
TITLE:
}

\section{Wave Emission from Heterogeneities Opens a Way to Controlling Chaos in the Heart}

\section{$\operatorname{AUTHOR}(S)$ :}

Pumir, A.; Nikolski, V.; Hörning, M.; Isomura, A.; Agladze, K.; Yoshikawa, K.; Gilmour, R.; Bodenschatz, E.; Krinsky V.

\section{CITATION:}

Pumir, A. ... [et al]. Wave Emission from Heterogeneities Opens a Way to Controlling Chaos in the Heart. Physical Review Letters 2007, 99: 208101

\section{ISSUE DATE:}

2007-11

URL:

http://hdl.handle.net/2433/49184

\section{RIGHT:}

Copyright (c) 2007 American Physical Society 


\title{
Wave Emission from Heterogeneities Opens a Way to Controlling Chaos in the Heart
}

\author{
A. Pumir, ${ }^{1, *}$ V. Nikolski, ${ }^{2}$ M. Hörning, ${ }^{3}$ A. Isomura, ${ }^{3}$ K. Agladze,${ }^{3, \dagger}$ K. Yoshikawa, ${ }^{3}$ R. Gilmour, ${ }^{4}$ \\ E. Bodenschatz, ${ }^{5}$ and V. Krinsky ${ }^{1,3}$ \\ ${ }^{1}$ Institut Non Linéaire de Nice, CNRS,F-06560 Valbonne, France \\ ${ }^{2}$ Medtronic Inc., 7000 Central Avenue Nebraska, MS CW330 Fridley Minnesota 55432-3576, USA \\ ${ }^{3}$ Department of Physics, Graduate School of Science, Kyoto University, Kyoto 606-8502, Japan \\ ${ }^{4}$ Department of Biomedical Sciences, T7 012C VRT, Cornell University, Ithaca, New York 14853-6401, USA \\ ${ }^{5}$ MPI for Dynamics and Self-Organization, Am Fassberg 17, D-37077 Göttingen, Germany
}

(Received 17 March 2007; published 13 November 2007)

\begin{abstract}
The effectiveness of chaos control in large systems increases with the number of control sites. We find that electric field induced wave emission from heterogeneities (WEH) in the heart gives a unique opportunity to have as many control sites as needed. The number of pacing sites grows with the amplitude of the electric field. We demonstrate that WEH has important advantages over methods used in clinics, and opens a new way to manipulate vortices in experiments, and potentially to radically improve the clinical methods of chaos control in the heart.
\end{abstract}

PACS numbers: 87.19.Hh, 87.50.Rr

Scattering and re-emission of waves by heterogeneities, induced by an acoustic or electromagnetic field, provides an important tool to investigate and control physical systems. We show that wave emission from heterogeneities (WEH) induced by periodic pulses of an electric field provides a powerful method of terminating chaos in the heart.

In a normal heart, regular waves of electric depolarization of the cellular membrane propagate to trigger the mechanical contractions. Cardiac chaos during lifethreatening arrhythmias is typically initiated by highfrequency rotating waves (spirals). The methods used in clinics are based either on a high intensity electric shock (defibrillation, $\sim 4000 \mathrm{~V}, 15 \mathrm{~A}$ when applied through the body), or on trains of low amplitude pulses (anti tachycardia pacing, ATP). Both methods have drawbacks: defibrillation shocks are potentially damaging; ATP fails to terminate high-frequency arrhythmias and developed chaos (fibrillation) [1].

Defibrillation requires a very high energy because it aims at terminating all waves indiscriminately. An approach to selectively terminate only rotating waves [2,3] involves unpinning a rotating wave anchored to an anatomical heterogeneity by applying a properly phased electric field pulse. Experiments show that this method requires an energy $\sim 20$ times smaller than a defibrillation shock [4]. But only a single rotating wave can be removed because distinct spirals have different phases.

It is well known that the effectiveness of chaos control in big systems increases with the number of control electrodes [5-9]. But connecting many electrodes is damaging to the heart. We found that WEH allows us to overcome this problem. The key feature is that the number and position of WEH wave emitting sites can be regulated by the electric field. This makes WEH capable of terminating multiple rotating waves, and opens new possibilities for termination of high-frequency tachycardias and fibrilla- tion, contrary to ATP. The mechanism used by WEH is pacing (from multiple sites). So the pulse energy required is just the energy needed for initiating excitation locally; it could be several orders of magnitude less than that needed for defibrillation.

It is likely that defibrillation terminates all propagating waves by using the same mechanism of WEH, but with a single high-energy pulse recruiting many spatial heterogeneities, down to very small scales. In this sense, defibrillation is a limiting case of WEH with only one pulse, and a very high energy.

WEH is based on the fact that an electric field changes the membrane potential of cardiac cells near conductivity heterogeneities. This phenomenon is known in cardiology as virtual electrodes or secondary sources, e.g., see Refs. [10-14]. The depolarization induced by a uniform electric field can be computed by solving the equation, obtained by linearizing the full cardiac equations around the resting potential, $V_{\text {rest }}$ :

$$
\nabla^{2} e-\frac{e}{\lambda^{2}}=0
$$

where $e$ is the difference between the membrane potential, $V$, and the resting potential, $V_{\text {rest }}$, and $\lambda$, the electrotonic length, is of order $\lambda \sim 1 \mathrm{~mm}$. Equation (1) must be solved with the boundary conditions: $e(\mathbf{r}) \rightarrow 0$ when $|\mathbf{r}| \rightarrow \infty$ and $\nabla(e+E \cdot \mathbf{r}) \cdot \mathbf{n}=0$ on the boundary of the obstacle, where $E$ is the applied electric field. For details in the derivation of the reduced model and boundary conditions, see [2]. A more precise calculation using the bidomain approach [10] would lead to qualitatively similar results (see $[2,3])$.

The solution can be explicitly determined in the case of a circular obstacle of radius $R$ :

$$
e(\mathbf{r})=-\frac{K_{1}(r / \lambda)}{K_{1}^{\prime}(R / \lambda)} E \lambda \cos \theta
$$


where $(r, \theta)$ are the coordinates of $\mathbf{r}$ in a polar coordinate system. The largest depolarization (largest value of $e$ ) is located on the circle: $e_{\operatorname{Max}}=-K_{1}(R / \lambda) / K_{1}^{\prime}(R / \lambda) E \lambda$. It is seen [Fig. 1(f)] that the smaller the obstacle size $R$, the smaller is the induced voltage $e$.

This observation suggests a way to control the number of wave emitting sites by changing the amplitude $E$ of electric field. A heterogeneity can emit a wave only if the voltage $e$ induced by an external electric field exceeds the excitation threshold $e_{\mathrm{th}}$. Thus, increasing the applied field $E$ results in increasing the number of wave emitting sites in cardiac tissue, where heterogeneities of different sizes are present. Obstacles of size much smaller than $\lambda$ trigger wave formation only for very high applied electric field, whereas larger size obstacles emit waves with a small electric field (much smaller than the defibrillation threshold).

To check these ideas, we performed numerical simulations of the cardiac equations, using the Luo-Rudy (LR) model [15] describing the membrane potential, $e$, and ionic currents in the cardiac cell. The parameters were modified as in [16].

The partial differential equations were solved in a 2-dimensional domain with no flux boundary conditions, with a second order Runge-Kutta integrator; the time step is $\Delta t=0.05 \mathrm{~ms}$. Finite differences are used; the mesh size is $\Delta x=0.225 \mathrm{~mm}$. The coupling heterogeneities are rep-
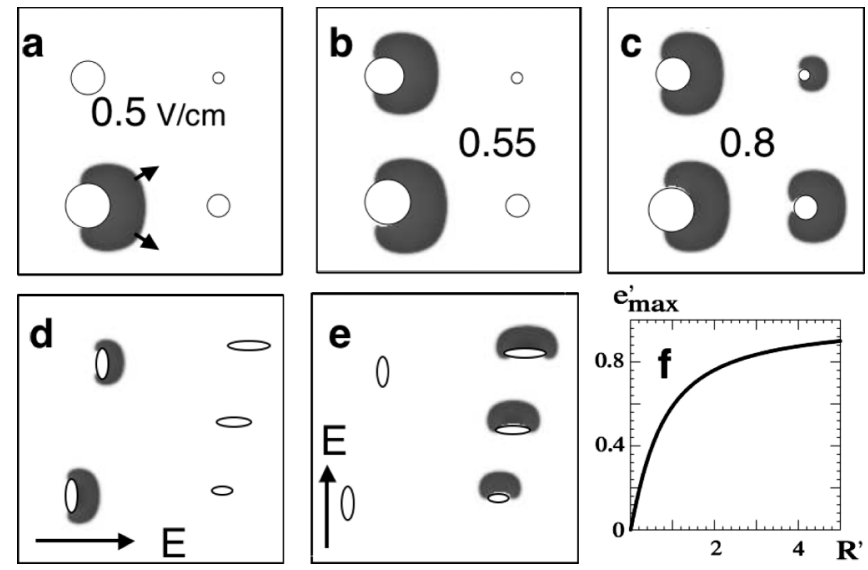

FIG. 1. Modifying the number and positions of pacing sites by changing the electric field. Obstacles are shown as white circles and ellipses. $(\mathrm{a}-\mathrm{c})$ The electric field is horizontal and increases from (a) to (c). (a) A field $E=0.5 \mathrm{~V} / \mathrm{cm}, 10 \mathrm{~ms}$ duration results in wave emission from 1 site. The short arrows indicate direction of the wave propagation. (b) With $E=0.55 \mathrm{~V} / \mathrm{cm}$, waves are emitted from 2 sites. (c) With $E=0.8 \mathrm{~V} / \mathrm{cm}$, waves are emitted from 4 sites. (d-e) The electric field changes direction from horizontal (d) to vertical (e), as indicated by the arrows. (d) $E_{X}=0.55 \mathrm{~V} / \mathrm{cm}, E_{Y}=0$. (e) $E_{X}=0, E_{Y}=0.55 \mathrm{~V} / \mathrm{cm}$. Note that wave emitting sites are different in (d) and (e). (f) Potential change $e_{\max }^{\prime}$ vs radius $R^{\prime}$ of a circular obstacle. Dimensionless coordinates: $e_{\max }^{\prime}=e_{\max } / E_{0} \lambda, R^{\prime}=R / \lambda, \lambda=$ $0.08 \mathrm{~cm}$ is the electrotonic constant. Simulations done in the LR model. resented by domains of simple shape (circular or elliptic), where the coupling, and hence the diffusion constant, are very much reduced: $D_{\text {in }}=D / 1000$, where $D=1 \mathrm{~cm}^{2} / \mathrm{s}$ is the diffusion constant in the medium. The sharp discontinuity in the diffusion coefficient effectively leads to the boundary condition at the surface of the obstacles discussed above. Trains of electric field pulses $10 \mathrm{~ms}$ duration were applied. Other details can be found in [2,3].

Figure 1 illustrates modifying the number and positions of pacing sites by changing the electric field. The tissue shown in Figs. 1(a) and 1(c) contains four circular obstacles, of radii $0.32 \mathrm{~cm}, 0.24 \mathrm{~cm}, 0.16 \mathrm{~cm}$, and $0.12 \mathrm{~cm}$. The smallest field $[E=0.5 \mathrm{~V} / \mathrm{cm}$, Fig. 1(a) $]$ induces a wave from the largest heterogeneity only. A slightly higher field, $E=0.55 \mathrm{~V} / \mathrm{cm}$, triggers a wave from the two larger obstacles, Fig. 1(b). At $E=0.8 \mathrm{~V} / \mathrm{cm}$, waves are generated from the 4 heterogeneities.

The effect of the electric field on obstacles of elongated shapes is illustrated in Figs. 1(d) and 1(e). The tissue contains 5 elliptical obstacles. Their long axes are vertical with 2 ellipses and horizontal with 3 ellipses. The semi axes of the ellipses $(a, b)=(0.24 \mathrm{~cm}, 0.07 \mathrm{~cm})$ and $(0.18 \mathrm{~cm}, 0.08 \mathrm{~cm})$ on the left of the picture, and $(a, b)=$ $(0.16 \mathrm{~cm}, 0.06 \mathrm{~cm}),(0.22 \mathrm{~cm}, 0.08 \mathrm{~cm})$, and $(0.28 \mathrm{~cm}$, $0.07 \mathrm{~cm}$ ) on the right side of the picture. We find that applying an horizontal (vertical) electric field of $E=$ $0.55 \mathrm{~V} / \mathrm{cm}$ for $10 \mathrm{~ms}$, see Figs. 1(d) and 1(e), leads to wave emissions from the ellipses with their long axes vertically (horizontally) oriented.

For an ellipse, the depolarization can be completely determined in the limit $\lambda \rightarrow \infty$. Indeed, in this case, Eq. (1) reduces to the Laplace equation, which can be solved by conformal transformations. Consider an ellipse $E$ of semiaxes $a$ and $b$, with an angle $\psi$ between the $x$-axis and the semiaxis $a$. Without any loss of generality, we take the electric field parallel to the $x$-axis. The conformal transformation,

$$
z=\exp (i \psi)\left(\zeta+\frac{\mu^{2}}{\zeta}\right)
$$

with $\mu=\sqrt{\left(a^{2}-b^{2}\right)} / 2$, maps the circle of radius $(a+$ $b) / 2$ in the $\zeta$-plane into the ellipse $E$ in the $z$-plane. The solution for $e$ reads

$$
e(z)=\operatorname{Re}\left(\frac{(a+b)^{2} \exp (-i \psi)-\left(a^{2}-b^{2}\right) \exp (i \psi)}{4 \zeta}\right)
$$

The largest value of $e$ is located on the ellipse $[\zeta=$ $\exp (i \theta) \times(a+b) / 2]$, at an angle given by $\tan \theta=-\frac{b}{a} \times$ $\tan \psi: \quad e_{\operatorname{Max}}=-E\left[\left(a^{2}+b^{2}\right) / 2+\left(b^{2}-a^{2}\right) / 2 \cos (2 \psi)\right]^{1 / 2}$. When $\psi=0$ (electric field parallel to the large axis), $e_{\text {Max }}(\psi=0)=E b$, whereas when $\psi=\pi / 2$, (the electric field parallel to the small semiaxis), $e_{\mathrm{Max}}=E a$.

The strong dependence of the maximum value of the depolarization on the angle diminishes when the value of $\lambda$ 
decreases. In the limit $\lambda \rightarrow 0$, an elementary boundary layer calculation shows that the largest value of the depolarization is $e_{\mathrm{Max}}=E \lambda$, independent of the shape and of the orientation of the obstacle. Figure 1 demonstrates that (i) increasing the intensity of the electric field leads to wave emission from an increasingly large number of heterogeneities, and (ii) changing the orientation of the field leads to wave emission from different heterogeneities.

These results suggest to use WEH as a method of chaos control of waves in the heart. It is more effective than ATP and requires much less energy than the defibrillating shock.

ATP cannot suppress high-frequency arrhythmias $(T<$ $250 \mathrm{~ms}$ ) [1]. This fact has a simple physical origin. If the frequency of pacing waves is below the frequency of a wave source, the pacing waves cannot reach the wave source [17] since excitable waves annihilate under collisions. So, high-frequency pacing waves are needed to terminate high-frequency arrhythmias. But the highfrequency waves cannot propagate through heterogeneities with an increased restoration time ("refractoriness"): every third or second wave is blocked (decayed), a phenomenon known as Wenckebach rhythm transformation [18]. This effect leads to a quasiperiodic modulation of the size of the entrained domain at high frequency.

WEH is a way to overcome these limitations. It is well known that the closer a pacing site is situated to a (pathological) wave source, the easier it can be eliminated. WEH permits to deliver pacing from many pacing sites spread all over the tissue. The number of pacing sites is controlled by the field intensity. This makes possible wave emission from heterogeneities located close to the core of any rotating wave, contrary to ATP, which uses a fixed pacing electrode.

Thus, WEH can suppress high-frequency cardiac arrhythmias contrary to conventional ATP, Fig. 2. Rotating waves were initiated in a 2-dimensional slab of tissue size $5.85 \mathrm{~cm} \times 6.75 \mathrm{~cm}$ containing multiple heterogeneities: a horizontal stripe (of width $0.45 \mathrm{~cm}$ ) where $G_{K}=$ $0.03525 \mathrm{mS} / \mu \mathrm{F}$, leading to a refractory period larger than the period of rotating waves, and 2 big and 6 small obstacles with radii: $3 \mathrm{~mm}$ (upper left); $3.6 \mathrm{~mm}$ (lower left); the small obstacles are $1 \mathrm{~mm}$. Pulses of electric field $10 \mathrm{~ms}$ duration were delivered with period $T=98 \mathrm{~ms}$. Electric field $E=1.1 \mathrm{~V} / \mathrm{cm}$ induced waves from big and small obstacles, Figs. 2(A)(2-3), and terminated all rotating waves, Fig. 2(A)(4). In contrast, with $E=0.6 \mathrm{~V} / \mathrm{cm}$, waves were induced only from 2 big obstacles, Figs. 2(B)(2,3) and rotating waves were not terminated, Fig. 2(B)(4). Fourier spectra, Fig. 2(C), show the underlying mechanism: the amplitude of the pacing frequency in the medium was much higher when many pacing sites were involved. WEH is thus better than ATP which uses one pacing site only.

Initiation of WEH waves in a more realistic model of heart muscle, cardiac myocytes in cell culture, is shown in Fig. 3(A). Rat cardiac myocytes were prepared as in [19] to

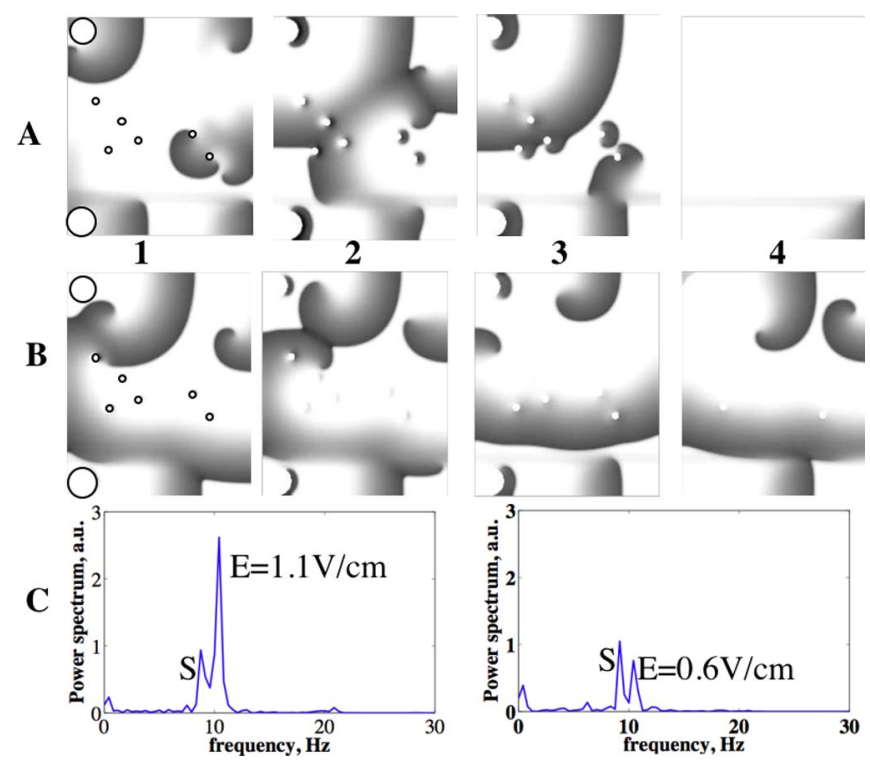

FIG. 2 (color online). Termination of rotating waves by WEH. (A) Electric field $E=1.1 \mathrm{~V} / \mathrm{cm}$. Rotating waves are removed. (A1) $t=0.795 \mathrm{~s}:$ Several rotating waves are seen. (A2) $t=0.85 \mathrm{~s}$ and (A3) $t=1.155 \mathrm{~s}$ : Pacing waves initiated from 2 big and 6 small obstacles by electric field pulses $10 \mathrm{~ms}$ duration are seen. (A4) $1.98 \mathrm{~s}$ : All rotating waves are terminated. (B) Electric field $E=0.6 \mathrm{~V} / \mathrm{cm}$. Rotating waves are not removed. (B1) $t=0.750 \mathrm{~s}$ : Two rotating waves are seen. (B2) $t=0.850 \mathrm{~s}$ and (B3) $t=1.155 \mathrm{~s}$ : Seen that pacing waves are initiated only from two big obstacles but not from 6 small obstacles. (B4) $t=2.040 \mathrm{~s}$ : Two rotating waves are still seen. The arrhythmia is not terminated. (A1), (B1) also show obstacles. (C) Power spectra of the pseudo ECG. Peak $S$ is induced by rotating waves, frequency $\sim 9 \mathrm{~Hz}$. Peak $E$ is induced by electric field pulses $10 \mathrm{~ms}$ duration with pacing frequency $f=$ $10.2 \mathrm{~Hz}$. In all figures, period of pacing $T=98 \mathrm{~ms}$. Meandering spirals, rotation time $T_{s} \sim 110-115 \mathrm{~ms}$.

form a cell layer $22 \mathrm{~mm}$ diameter. WEH excitation waves were induced by electric field pulses and imaged using the fluorescent $\mathrm{Ca}_{i}$ indicator Fluo-4. Comparing Figs. 3 $(\mathrm{A}, \mathrm{B}, \mathrm{C})$ permits us to identify the naturally existing heterogeneities where the WEH waves were initiated; they are very different from oversimplified obstacles in Fig. 2. Figure 3(B) shows mechanism of termination of a rotating wave by WEH wave trains: every pacing wave induces a shift of the wave tip, and when the tip reaches the medium boundary, the rotating wave is terminated. The experiment demonstrates that the electric field needed for WEH termination of a rotating wave is below the defibrillation threshold for this preparation. One single electric field pulse (corresponding to a defibrillation shock) never terminates the rotating wave while the train of pulses can.

Quantitative estimates can be obtained by comparing with experimental data. Unpinning [3] requires an electric filed $E \sim 1.2 \mathrm{~V} / \mathrm{cm} \mathrm{[4],} \sim 5$ times smaller than the defibrillation field. Unpinning needs the induced wave to propagate in one direction only. For this, the stimulus should be 


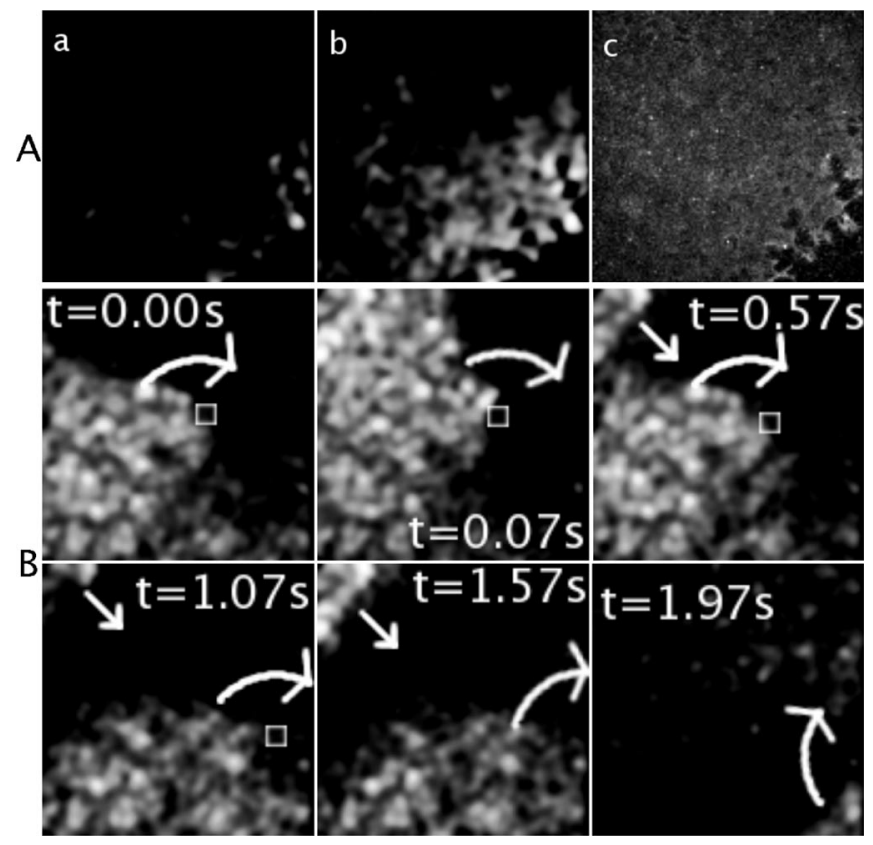

FIG. 3. WEH waves in cultured cardiac myocytes. (A) WEH waves. Initiation (a) and propagation (b). A pulse $1 \mathrm{~V} / \mathrm{cm}, 9 \mathrm{~ms}$ duration was delivered before frame (a). (c) shows the structure of the cell layer (transmitted light), where the heterogeneities are seen in the lower right corner. (B) Termination of a rotating wave by $1.5 \mathrm{~V} / \mathrm{cm}$ pulses. $t=0$. A rotating wave is shown. A curved arrow indicates the rotation direction. A small white square indicates wave tip position. Waves are induced by the far field pulses, from an heterogeneity not seen in the field of view. They propagate from the left upper corner, in the direction shown by the rectilinear arrow at $t=0.57 \mathrm{~s}, 1.07 \mathrm{~s}, 1.57 \mathrm{~s}$. Every of them induces a shift of the wave tip (white square) to the right, and it disappears from the field of view at $t=1.57 \mathrm{~s}$. All figures show a field of view of $2 \mathrm{~mm} \times 2 \mathrm{~mm}$.

above the Maxwell level $E_{M}$ (in two variable models [20]), or above the lower level of vulnerability (LLV) in cardiac tissue, while WEH requires a stimulus only above the excitation threshold $E_{\text {ex }}$. In cardiac tissue, the excitation threshold $E_{\mathrm{ex}}$ is 5-10 times smaller than LLV [21]. Thus, the pulse energy WEH may be 25-100 less than needed for unpinning (energy is proportional to $E^{2}$ ) and the electric field $\sim 0.25-0.12 \mathrm{~V} / \mathrm{cm}$. Since the defibrillation field is $\sim 5.6 \mathrm{~V} / \mathrm{cm}$, this means 500-2000 times less pulse energy. This is a simplified upper estimate; real gain may be an order of magnitude less since the pacing threshold increases with increasing the pacing frequency. Only experiments with real hearts can precisely determine the gains in energy.

In summary, wave emission from heterogeneities (WEH) in the heart opens a way for a multisite pacing that was not possible with conventional methods. This permits us to pace tissue at high frequencies, which was not possible with standard antitachycardia pacing (ATP). WEH is not limited to one reentry only, as for unpining [2-4]. This may result in a new way to control vortices and chaos in the heart.

We have benefited from discussions with S. Sinha, and acknowledge support of KITP, NIH No. HL073644, JSPS (Project No. 18GS0421), and IFCPAR under Project No. 3404-4.

*alain.pumir@unice.fr

${ }^{\dagger}$ Spatio-Temporal Project, JST

[1] M.S. Wathen et al., Circulation 110, 2591 (2004).

[2] A. Pumir and V. Krinsky, J. Theor. Biol. 199, 311 (1999).

[3] S. Takagi et al., Phys. Rev. Lett. 93, 058101 (2004).

[4] C. Ripplinger et al., Am J. Physiol. 291, H184 (2006).

[5] M. Allessie et al., Circulation 84, 1689 (1991).

[6] I. Aranson, H. Levine, and L. Tsimring, Phys. Rev. Lett. 72, 2561 (1994).

[7] H. Gang and Q. Zhilin, Phys. Rev. Lett. 72, 68 (1994).

[8] W. J. Rappel, F. Fenton, and A. Karma, Phys. Rev. Lett. 83, 456 (1999).

[9] S. Sinha, A. Pande, and R. Pandit, Phys. Rev. Lett. 86, 3678 (2001).

[10] N.G. Sepulveda, B.J. Roth, and J.P. Wikswo, Jr., Biophys. J. 55, 987 (1989).

[11] E. A. Sobie et al., Biophys. J. 73, 1410 (1997).

[12] M. G. Fishler, J. Cardiovasc. Electrophysiol. 9, 384 (1998).

[13] V. Fast et al., Circ. Res. 82, 375 (1998).

[14] M. C. Woods et al., Heart Rhythm 3, 751 (2006).

[15] C. Luo and Y. Rudy, Circ. Res. 68, 1501 (1991).

[16] F. Xie et al., Am. J. Physiol. 280, H535 (2001).

[17] V. Krinsky, Systems Theory Research 20, 46 (1970).

[18] C. B. Upshaw Jr. et al., Ann. Intern. Med. 130, 158 (1999).

[19] K. Agladze et al., Am J. Physiol. 293, H503 (2007).

[20] C. F. Starmer et al., Biophys. J. 65, 1775 (1993).

[21] A. W. Hamer et al., J. Am. Coll. Cardiol. 3, 751 (1984). 